\title{
A NEW METHOD TO SYNTHESIZE 3,5-DIARYLPYRAZOL DERIVATIVES
}

\author{
XIANQIANG HUANG, JIANMIN DOU ${ }^{1}$ DACHENG LI DAQI WANG
}

Department of Chemistry \& Chemical Engineering, Liaocheng University Liaocheng, 252059, China

(Received 26 February 2008 - Accepted 22 July 2008)

\begin{abstract}
Twelve 3, 5-diarylpyrazoles have been synthesized by microwave-assisted condensation reaction in a short and concise manner using various aromatic

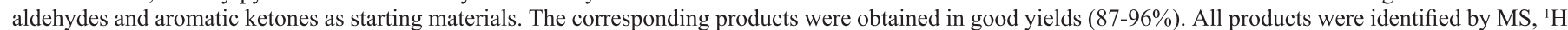

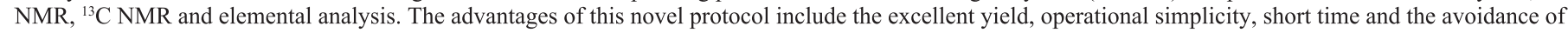
the use of expensive catalysts.
\end{abstract}

Keywords: 2, 4, 6-Triarylpyrylium salts, 3,5-Diarylpyrazoles, Microwave irradiation.

\section{INTRODUCTION}

Pyrazoles are important nitrogen-containing five-membered heterocyclic compounds ${ }^{1-3}$ and numerous compounds containing pyrazole have been shown to exhibit antihyperglycemic, analgesic, anti-inflammatory, antipyretic, antibacterial activity ${ }^{4}$. Moreover, metal complexation to the pyrazole heterocycle could offer a straightforward way to couple the effect of a metal bound to one heterocyclic nitrogen with the ability of the $\mathrm{N}-\mathrm{H}$ to donate a proton or hydrogen bond ${ }^{5}$. Thus, continuous efforts have been devoted to the development of more general and versatile synthetic methodologies to this class of compounds ${ }^{6-7}$.

Meanwhile, high-speed synthesis under microwave irradiation conditions has attracted a considerable amount of attention ${ }^{8-9}$, and some important reviews in the study of microwave assisted organic synthesis have been published ${ }^{10}$, the scope of applications is very extended and concerns a wide spectrum of organic synthesis procedures including, for instance, heterocyclic, organometallic, radio photo and combinatorial chemistries ${ }^{11-12}$.

In recent years, Wang et al have developed a general synthesis of 2,4,6triarylpyridiums, which were important oxygen-containing building blocks and their use in heterocyclic preparations (e.g., pyridines, thiophenes, furans and diazepines) has been extensively described ${ }^{13-14}$.

In continuation of our efforts to develop novel synthetic routes for carboncarbon and carbon-heteroatom bond formations and heterocycles using 2,4,6triarylpyridiums as starting materials, we have been interested in developing new, more efficient, and synthetically useful reactions for the synthesis of biologically active molecules via microwave acceleration Thus, the aim of this work is to demonstrate the advantages obtained by the use of microwave irradiation for the synthesis of 3,5-diarylpyrazoles.

\section{EXPERIMENTAL}

${ }^{1} \mathrm{H}$ NMR spectra $(200 \mathrm{MHz})$ were recorded using a Bruker AC-E 200 $\mathrm{MHz}, 400 \mathrm{MHz}$ spectrometer in $\mathrm{CDCl}_{3}, \mathrm{CD}_{3} \mathrm{COCD}_{3}$, DMSO-d $d_{6}$ with TMS as an internal standard. Mass spectra measurements were performed on a QP-1000A GC-MS spectrometer by EI ionization at $70 \mathrm{eV}$. Purification of products was performed via flash chromatography with 200-400 mesh silica gel [petroleum ether $\left(\mathrm{bp} 60-90^{\circ} \mathrm{C}\right)-\mathrm{EtOAc}, 5: 1$ ]. The chemicals were obtained from commercial sources.

General experimental procedure

We prepared a series of pyrylium salts in the absence of solvent under microwave irradiation. Then, 1,3,5-triphenyl-2-pentene-1,5-diketone were prepared by the reaction of pyrylium salts with $\mathrm{NaOAc}$. Next, 1,3,5-triphenyl2-pentene-1,5-diketone $(0.1 \mathrm{mmol})$ was added in an open flask, followed by the addition of hydrazine hydrate $(1 \mathrm{mmol})$ and acetic acid $(1.2 \mathrm{ml})$. Then the mixture was irradiated in microwave (375W) in an open flask for $5 \mathrm{~min}$. After the reaction was completed, the reaction mixture was left to room temperature, then water was added obtaining a white precipitate, the solid was isolated by filtration and washed with water. The product was purified by recrystallization once or twice from dilute ethanol or by flash chromatography with $200-400$ mesh silica gel [petroleum ether (bp 60-90 ${ }^{\circ} \mathrm{C}$ )-EtOAc, 5:1]. The reactions are shown in Scheme 1 and the results are summarized in Table 1

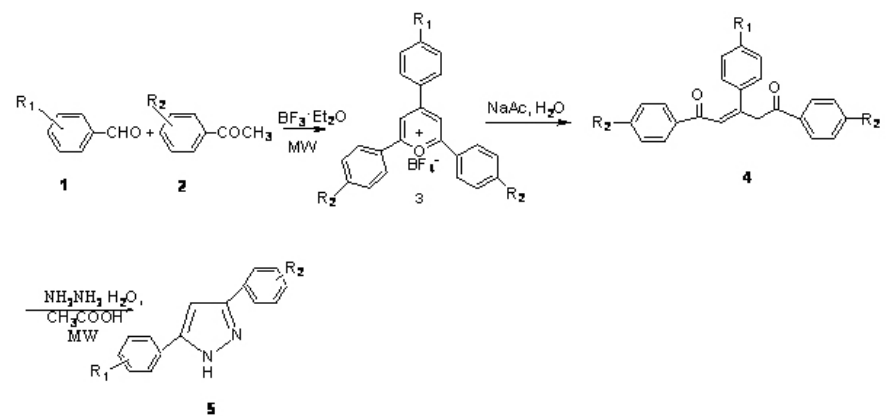

Scheme 1. The synthetic route of the title compounds.

Table 1. Synthesis of 3, 5-diarylpyrazoles.

\begin{tabular}{|c|c|c|c|c|}
\hline Entry & $\mathrm{R}^{1}$ & $\mathrm{R}^{2}$ & Yield*(\%) & Time(min)/power(W) \\
\hline $\mathbf{5 a}$ & $\mathrm{H}$ & $\mathrm{H}$ & 96 & $5 / 375$ \\
\hline $\mathbf{5 b}$ & $\mathrm{H}$ & $4-\mathrm{CH}_{3}$ & 97 & $5 / 375$ \\
\hline $\mathbf{5 c}$ & $\mathrm{H}$ & $4-\mathrm{Cl}$ & 89 & $6 / 375$ \\
\hline $\mathbf{5 d}$ & $\mathrm{H}$ & $4-\mathrm{Br}$ & 94 & $7 / 375$ \\
\hline $\mathbf{5 e}$ & $4-\mathrm{CH}_{3}$ & $\mathrm{H}$ & 95 & $5 / 375$ \\
\hline $\mathbf{5 f}$ & $4-\mathrm{CH}_{3}$ & $4-\mathrm{CH}_{3}$ & 91 & $5 / 375$ \\
\hline $\mathbf{5 g}$ & $4-\mathrm{CH}_{3}$ & $4-\mathrm{Cl}$ & 90 & $5 / 375$ \\
\hline $\mathbf{5 h}$ & $4-\mathrm{CH}_{3}$ & $4-\mathrm{Br}$ & 92 & $5 / 375$ \\
\hline $\mathbf{5 i}$ & $4-\mathrm{Cl}$ & $\mathrm{H}$ & 88 & $6 / 375$ \\
\hline $\mathbf{5 j}$ & $4-\mathrm{Cl}$ & $4-\mathrm{CH}$ & 93 & $6 / 375$ \\
\hline $\mathbf{5 k}$ & $4-\mathrm{Cl}$ & $4-\mathrm{Cl}$ & 92 & $7 / 375$ \\
\hline $\mathbf{5 l}$ & $4-\mathrm{Cl}$ & $4-\mathrm{Br}$ & 87 & $7 / 375$ \\
\hline
\end{tabular}

*The yield is based on the compound 4

Taking the reaction of 1,3,5-triphenyl-2-pentene-1,5-diketone with hydrazine hydrate as an example, we investigated the effect of the power and time of microwave irradiation. The results are summarized in the Table 2 and Table 3. The results shown that the title compounds can rapidly be obtained in $375 \mathrm{~W}$ power for $5 \mathrm{~min}$. 
Table 2. Effect of the power of MWI on synthesis of 3, 5-diarylpyrazoles.

\begin{tabular}{|c|c|c|c|c|c|}
\hline Power (W) & 150 & 225 & 300 & 375 & 450 \\
\hline Yield (\%) & 46 & 72 & 85 & 96 & 92 \\
\hline
\end{tabular}

Table 3. Effect of the time of MWI on synthesis of 3, 5-diarylpyrazoles.

\begin{tabular}{|c|c|c|c|c|c|}
\hline Time (min) & 2 & 3 & 4 & 5 & 6 \\
\hline Yield (\%) & 58 & 75 & 90 & 96 & 96 \\
\hline
\end{tabular}

From the tables, we found the optimum condition of the reaction: the time of MWI is $5 \sim 7 \mathrm{~min}$, the power of MWI is $375 \mathrm{~W}$.

Spectral data for compounds

3,5-diphenyl-1H-pyrazole

White crystals, mp $199-201^{\circ} \mathrm{C}\left(\right.$ lit. $\left.{ }^{[15]} 199^{\circ} \mathrm{C}\right),{ }^{1} \mathrm{H}$ NMR $\left(200 \mathrm{MHz}, \mathrm{CDCl}_{3}\right)$ $\delta=7.75-7.69(\mathrm{~m}, 5 \mathrm{H}), 7.45-7.24(\mathrm{~m}, 5 \mathrm{H}), 6.84(\mathrm{~s}, 1 \mathrm{H}),{ }^{13} \mathrm{C} \mathrm{NMR}(100 \mathrm{MHz}$, $\left.\mathrm{CDCl}_{3}\right) \delta=148.75,130.99,128.90,128.42,125.66,100.22 ; \mathrm{MS}(\mathrm{m} / \mathrm{e}, \%)$ : $220\left(\mathrm{M}^{+}, 80.38\right), 191\left(\mathrm{M}^{+}-\mathrm{N}-\mathrm{NH}, 13.87\right), 77\left(\mathrm{C}_{6} \mathrm{H}_{5}, 32.16\right)$; Anal Calcd for $\mathrm{C}_{15} \mathrm{H}_{12} \mathrm{~N}_{2}: \mathrm{C}, 81.79 ; \mathrm{H}, 5.49 ; \mathrm{N}, 12.72$; Found: $\mathrm{C}, 81.88 ; \mathrm{H}, 5.163 ; \mathrm{N}, 12.92$.

\section{5-phenyl-3-p-tolyl-1H-pyrazole}

White crystals, mp $179-181^{\circ} \mathrm{C}\left(\right.$ lit. $\left.^{[16]} 183-184^{\circ} \mathrm{C}\right){ }^{1} \mathrm{H}$ NMR $(200 \mathrm{MHz}$, $\left.\mathrm{CD}_{3} \mathrm{COCD}_{3}\right) \delta=12.59(\mathrm{~b}, 1 \mathrm{H}), 8.01-7.36(\mathrm{~m}, 9 \mathrm{H}), 7.19(\mathrm{~s}, 1 \mathrm{H}), 2.47(\mathrm{~s}, 3 \mathrm{H})$; ${ }^{13} \mathrm{C}$ NMR $\left(100 \mathrm{MHz}, \mathrm{CDCl}_{3}\right) \delta=149.19,148.06,138.28,129.56,128.82$, $128.23,125.64,125.52,99.86,21.28$; MS (m/e, \%): $234\left(\mathrm{M}^{+}, 100\right), 205\left(\mathrm{M}^{+}-\mathrm{N}-\right.$ $\mathrm{NH}, 6.15), 91\left(\mathrm{C}_{6} \mathrm{H}_{5} \mathrm{CH}_{2}, 17.58\right), 77\left(\mathrm{C}_{6} \mathrm{H}_{5}, 62.10\right)$; Anal Calcd for $\mathrm{C}_{16} \mathrm{H}_{14} \mathrm{~N}_{2}: \mathrm{C}$, $82.02 ; \mathrm{H}, 6.024 ; \mathrm{N}, 11.95$; Found: C, 81.81; H, 5.833; N, 12.04 .

\section{3-(4-chlorophenyl)-5-phenyl-1H-pyrazole}

White crystals, mp $218-220^{\circ} \mathrm{C}\left(\right.$ lit $\left.^{[17]} 217^{\circ} \mathrm{C}\right) ;{ }^{1} \mathrm{H}$ NMR $\left(200 \mathrm{MHz}, \mathrm{CDCl}_{3}\right)$ $\delta=7.68-7.41(\mathrm{~m}, 9 \mathrm{H}), 6.83(\mathrm{~s}, 1 \mathrm{H}) ;{ }^{13} \mathrm{C}$ NMR $\left(100 \mathrm{MHz}, \mathrm{CD}_{3} \mathrm{COCD}_{3}\right) \delta=$ $149.19,148.06,138.28,129.66,128.84,127.70,126.12,100.52 ; \mathrm{MS}(\mathrm{m} / \mathrm{e}$, \%): $256\left(\mathrm{M}^{+}+2,34.86\right), 254\left(\mathrm{M}^{+}, 100\right), 225\left(\mathrm{M}^{+}-\mathrm{N}-\mathrm{NH}, 9.43\right), 111\left(\mathrm{ClC}_{6} \mathrm{H}_{5}\right.$, 48.48), $77\left(\mathrm{C}_{6} \mathrm{H}_{5}, 38.64\right)$; Anal Calcd for $\mathrm{C}_{15} \mathrm{H}_{11} \mathrm{ClN}_{2}$ : C,70.73; H, 4.35; N, 10.99; Found: C, 70.67; H, 4.28; N, 11.08 .

\section{3-(4-bromophenyl)-5-phenyl-1H-pyrazole}

White crystals, mp $217-218^{\circ} \mathrm{C}$ (lit $\left.{ }^{[17]} 215^{\circ} \mathrm{C}\right) ;{ }^{1} \mathrm{H} \mathrm{NMR}\left(200 \mathrm{MHz}, \mathrm{CDCl}_{3}\right.$ ) $\delta=7.66-7.35(\mathrm{~m}, 9 \mathrm{H}), 6.77(\mathrm{~s}, 1 \mathrm{H}), \mathrm{MS}(\mathrm{m} / \mathrm{e}, \%): 300\left(\mathrm{M}^{+}+2,93.68\right), 298\left(\mathrm{M}^{+}\right.$, 100), 269( $\left.\mathrm{M}^{+}-\mathrm{N}-\mathrm{NH}, 7.14\right), 77\left(\mathrm{C}_{6} \mathrm{H}_{5}, 16.49\right)$.

\section{3-phenyl-5-p-tolyl-1H-pyrazole}

White crystals, mp $180-182^{\circ} \mathrm{C}\left(\mathrm{lit}^{[16]} 183-184^{\circ} \mathrm{C}\right) ;{ }^{1} \mathrm{H}$ NMR $(200 \mathrm{MHz}$, $\left.\mathrm{CD}_{3} \mathrm{COCD}_{3}\right) \delta=12.54(\mathrm{~b}, 1 \mathrm{H}), 7.97-7.28(\mathrm{~m}, 9 \mathrm{H}), 7.14(\mathrm{~s}, 1 \mathrm{H}), 2.42(\mathrm{~s}, 3 \mathrm{H}) ;{ }^{13} \mathrm{C}$ NMR $\left(100 \mathrm{MHz}, \mathrm{CDCl}_{3}\right) \delta=149.08,148.35,138.22,131.39,129.53,128.80$, 128.19, 128.09, 125.65, 125.53, 99.84, 21.26; MS (m/e, \%): $234\left(\mathrm{M}^{+}, 100\right)$, 205( $\left.\mathrm{M}^{+}-\mathrm{N}-\mathrm{NH}, 6.15\right), 91\left(\mathrm{C}_{6} \mathrm{H}_{5} \mathrm{CH}_{2}, 17.58\right), 77\left(\mathrm{C}_{6} \mathrm{H}_{5}, 62.10\right)$; Anal Calcd for $\mathrm{C}_{16} \mathrm{H}_{14} \mathrm{~N}_{2}: \mathrm{C}, 82.02 ; \mathrm{H}, 6.024 ; \mathrm{N}, 11.95$; Found: $\mathrm{C}, 81.72 ; \mathrm{H}, 5.627 ; \mathrm{N}, 12.44$.

\section{3,5-dip-tolyl-1H-pyrazole}

White crystals, $\mathrm{mp} 237-238^{\circ} \mathrm{C} ;{ }^{1} \mathrm{H}$ NMR $\left(200 \mathrm{MHz}, \mathrm{CD}_{3} \mathrm{COCD}_{3}\right) \delta=12.39$ $(\mathrm{b}, 1 \mathrm{H}), 7.78-7.23(\mathrm{~m}, 8 \mathrm{H}), 7.02(\mathrm{~s}, 1 \mathrm{H}), 2.389(\mathrm{~s}, 6 \mathrm{H}) ;{ }^{13} \mathrm{C}$ NMR $(100 \mathrm{MHz}$, $\left.\mathrm{CDCl}_{3}\right) \delta=150.31,148.73,138.50,129.56,127.90,125.68,99.82,21.29$; MS (m/e, \%): $248\left(\mathrm{M}^{+}, 100\right), 233\left(\mathrm{M}^{+}-\mathrm{CH}_{3}, 2.35\right), 219\left(\mathrm{M}^{+}-\mathrm{N}-\mathrm{NH}, 3.71\right), 91$ $\left(\mathrm{C}_{6} \mathrm{H}_{5} \mathrm{CH}_{2}, 5.01\right), 77\left(\mathrm{C}_{6} \mathrm{H}_{5}, 9.64\right)$; Anal Calcd for $\mathrm{C}_{17} \mathrm{H}_{16} \mathrm{~N}_{2}: \mathrm{C}, 82.22 ; \mathrm{H}, 11.28$; $\mathrm{N}: 6.49$; Found: $\mathrm{C}, 81.88 ; \mathrm{H}, 10.60 ; \mathrm{N}, 6.06$.

\section{3-(4-chlorophenyl)-5-p-tolyl-1H-pyrazole}

White crystals, mp $236-237^{\circ} \mathrm{C} ;{ }^{1} \mathrm{H}$ NMR $\left(200 \mathrm{MHz}, \mathrm{CD}_{3} \mathrm{COCD}_{3}\right) \delta=12.70$ (b, $1 \mathrm{H}), 8.06-7.43(\mathrm{~m}, 8 \mathrm{H}), 7.23(\mathrm{~s}, 1 \mathrm{H}), 2.49(\mathrm{~s}, 3 \mathrm{H}) ;{ }^{3} \mathrm{C} \mathrm{NMR}(100 \mathrm{MHz}$, DMSO- $\left.d_{6}\right) \delta=149.23,148.54,137.34,132.07,131.68,129.43,128.78,127.05$, $126.75,125.03,120.63,99.52,20.83$; MS (m/e, \%): $270\left(\mathrm{M}^{+}+2,31.66\right), 268$ $\left(\mathrm{M}^{+}, 100\right), 233\left(\mathrm{M}^{+}-\mathrm{Cl}, 5.47\right), 111\left(\mathrm{ClC}_{6} \mathrm{H}_{5}, 5.70\right), 77\left(\mathrm{C}_{6} \mathrm{H}_{5}, 38.64\right)$; Anal Calcd for $\mathrm{C}_{16} \mathrm{H}_{13} \mathrm{ClN}_{2}$ : C, 71.50; H, 4.88; N, 10.42; Found: $\mathrm{C}, 71.34 ; \mathrm{H}, 4.63 ; \mathrm{N}$, 10.51 .

3-(4-bromophenyl)-5-p-tolyl-1H-pyrazole

White crystals, mp $244-245^{\circ} \mathrm{C}$; ${ }^{1} \mathrm{H}$ NMR $\left(200 \mathrm{MHz}, \mathrm{CDCl}_{3}\right) \delta=7.63-7.57$ $(\mathrm{m}, 8 \mathrm{H}), 6.79(\mathrm{~s}, 1 \mathrm{H}), 2.40(\mathrm{~s}, 3 \mathrm{H}) ;{ }^{3} \mathrm{C} \mathrm{NMR}\left(100 \mathrm{MHz}, \mathrm{DMSO}-\mathrm{d}_{6}\right) \delta=1149.07 \%$,
$148.14,137.35,132.07,131.69,129.44,128.79,127.06,126.75,125.04,99.53$, 20.83; MS (m/e, \%): $314\left(\mathrm{M}^{+}+2,100\right), 312\left(\mathrm{M}^{+}, 95.06\right), 283\left(\mathrm{M}^{+}-\mathrm{N}-\mathrm{NH}, 2.61\right)$, 233( $\mathrm{M}^{+}-\mathrm{Br}$, 9.26); Anal Calcd for $\mathrm{C}_{16} \mathrm{H}_{13} \mathrm{BrN}_{2}: \mathrm{C}, 61.39 ; \mathrm{H}, 4.18$; N, 8.94; Found: C, 61.30; H, 4.14; N, 8.93 .

\section{5-(4-chlorophenyl)-3-phenyl-1H-pyrazole}

White crystals, mp $216-218^{\circ} \mathrm{C}$ ( lit $\left.^{177} 217^{\circ} \mathrm{C}\right) ;{ }^{1} \mathrm{H}$ NMR $(200 \mathrm{MHz}$, $\left.\mathrm{CD}_{3} \mathrm{COCD}_{3}\right) \delta=12.65(\mathrm{~b}, 1 \mathrm{H}), 7.97-7.36(\mathrm{~m}, 9 \mathrm{H}), 7.19(\mathrm{~s}, 1 \mathrm{H}) ;{ }^{3} \mathrm{C}$ NMR $\left(100 \mathrm{MHz}, \mathrm{DMSO}-d_{6}\right) \delta=149.12,148.64,132.16,128.88,128.83,127.97$, 126.78, 125.11, 99.90; MS (m/e, \%): $256\left(\mathrm{M}^{+}+2,27.99\right), 254\left(\mathrm{M}^{+}, 100\right)$, 225( $\left.\mathrm{M}^{+}-\mathrm{N}-\mathrm{NH}, 7.43\right), 111\left(\mathrm{ClC}_{6} \mathrm{H}_{5}, 4.82\right), 77\left(\mathrm{C}_{6} \mathrm{H}_{5}, 43.21\right)$; Anal Calcd for $\mathrm{C}_{15} \mathrm{H}_{11} \mathrm{ClN}_{2}: \mathrm{C}, 70.73 ; \mathrm{H}, 4.35 ; \mathrm{N}, 10.99$; Found: $\mathrm{C}, 70.60 ; \mathrm{H}, 4.244 ; \mathrm{N}, 11.17$.

\section{5-(4-chlorophenyl)-3-p-tolyl-1H-pyrazole}

White crystals, $\mathrm{mp} 236-237^{\circ} \mathrm{C} ;{ }^{1} \mathrm{H}$ NMR $\left(200 \mathrm{MHz}, \mathrm{CD}^{\mathrm{COCD}}\right) \delta=12.29$ (s, 1H), 7.68-7.02 (m, 8H), $6.84(\mathrm{~s}, 1 \mathrm{H}), 2.37(\mathrm{~s}, 3 \mathrm{H}) ;{ }^{13} \mathrm{C}$ NMR $(100 \mathrm{MHz}$, DMSO- $d$ ) $\delta=149.23,148.62,132.07,129.44,128.79,126.75,125.03,99.53$, 20.83; MS (m/e, \%): $270\left(\mathrm{M}^{+}+2,35.21\right), 268\left(\mathrm{M}^{+}, 100\right), 239\left(\mathrm{M}^{+}-\mathrm{N}-\mathrm{NH}, 4.27\right)$, $111\left(\mathrm{ClC}_{6} \mathrm{H}_{5}, 23.03\right), 77\left(\mathrm{C}_{6} \mathrm{H}_{5}, 34.45\right)$; Anal Calcd for $\mathrm{C}_{16} \mathrm{H}_{13} \mathrm{ClN}_{2}$ : C, 71.50 ; H, 4.88; N ,10.42; Found: C,71.40; H, 4.67; N: 10.58 .

\section{3,5-bis(4-chlorophenyl)-1H-pyrazole}

White crystals, mp $248-249^{\circ} \mathrm{C}$; ${ }^{1} \mathrm{H}$ NMR $\left(200 \mathrm{MHz}, \mathrm{CDCl}_{3}\right) \delta=7.67-$ $7.38(\mathrm{~m}, 8 \mathrm{H}), 6.80(\mathrm{~s}, 1 \mathrm{H}) \cdot{ }^{3} \mathrm{C}$ NMR $(100 \mathrm{MHz}$, DMSO- $d) \delta=149.67,148.58$, 132.34, 128.89, 126.79, 100.23; MS (m/e, \%): $290\left(\mathrm{M}^{+}+2,68.23\right), 288\left(\mathrm{M}^{+}\right.$, 100), $259\left(\mathrm{M}^{+}-\mathrm{N}-\mathrm{NH}, 3.46\right), 111\left(\mathrm{ClC}_{6} \mathrm{H}_{5}, 8.63\right)$; Anal Calcd for $\mathrm{C}_{16} \mathrm{H}_{13} \mathrm{ClN}_{2}: \mathrm{C}$, $62.30 ; \mathrm{H}, 3.48 ; \mathrm{N}, 9.69$; Found: C,62.47; H, 3.223; N: 9.771.

\section{3-(4-bromophenyl)-5-(4-chlorophenyl)-1H-pyrazole}

White crystals, mp $248-250^{\circ} \mathrm{C}$; ${ }^{1} \mathrm{H}$ NMR $\left(200 \mathrm{MHz}, \mathrm{CDCl}_{3}\right) \delta=7.63-7.28$ $(\mathrm{m}, 8 \mathrm{H}), 6.82(\mathrm{~s}, 1 \mathrm{H}) ; \mathrm{MS}(\mathrm{m} / \mathrm{e}, \%): 334\left(\mathrm{M}^{+}+2,100\right), 332\left(\mathrm{M}^{+}, 76.43\right), 305$ $\left(\mathrm{M}^{+}-\mathrm{N}-\mathrm{NH}, 3.55\right), 111\left(\mathrm{ClC}_{6} \mathrm{H}_{5}, 8.21\right)$; Anal Calcd for $\mathrm{C}_{16} \mathrm{H}_{13} \mathrm{ClN}_{2}$ : C, $54.00 ; \mathrm{H}$, $3.02 ; \mathrm{N}, 8.39$; Found: C, $53.80 ; \mathrm{H}, 2.807 ; \mathrm{N}: 8.692$.

\section{CONCLUSIONS}

In summary, a benign, simple, versatile routes to 3, 5-diarylpyrazoles in high yield have been demonstrated. In order to investigate the microwave energy transfer system both mineral supports and a polar paste system were surveyed. We have found that acetic acid is a good catalyst in a new, microwave assisted method for the products synthesis. On the other hand, the most significant result remains on using a polar paste system. The excellent product yields and simple washing with water and filtration make this methodology as an alternative platform under the umbrella of environmentally green and safe processes. In general, these approaches lead to a clean, efficient and economical technology (green chemistry); safety is largely increased, workup is considerably simplified, cost is reduced. The extension of this type of reaction is actually under progress.

\section{ACKNOWLEDGEMENTS}

This work was financially supported by the Natural Science Foundation of China (No. 20671048) and the the National Natural Science Foundation of Liaocheng University (No. X051040).

\section{REFERENCES}

J. Elguero, P. Goya, N. Jagerovic, A. M. S. Silva, Targets Heterocycl. Syst. 6, 52, (2002).

S. W. Djuric, N. Y. BaMaung, A.Basha, H. Liu, J. R. Luly, D. J. Madar, R. J. Sciotti, N. P. Tu, F. L.Wagenaar, P. E. Wiedman, X. Zhou, S. Ballaron, J. Bauch, Y.- W. Chen, X. G. Chiou, T. Fey, D. Gauvin, E. Gubbins, G. C. Hsieh, K. C. Marsch, K. W. Mollison, M. Pong, T. K. Shaughnessy, M. P. Sheets, M. Smith, J. M.Trevillyan, U. Warrior, C. D. Wegner, G. W.Carter, J. Med. Chem. 43, 2975, (2000).

M. A. P. Martins, W. Cunico, C. M. P. Pereira, A. F. C. Flores, H. G. Bonacorso, N. Zanatta, Curr. Org. Synth. 1, 391, (2004),

S. G. Küçükgüzel, S. Rollas, H. Erdeniz, M. Kiraz, A. C. Ekinci, A. Vidin, Prog. Drug Res. 35, 761, (2000).

(a) Y. R. Huang, J. A. Katzenellenbogen, Org. Lett. 2, 2833,(2000). (b) X. J. Wang, J. Tan, K. Grozinger, R. Betageri, T. Kirrane, J. R. Proundfoot, Tetrahedron Lett. 41, 5321, (2000).

M. C. Bagley, M. C. Lubinu, C. Mason, Synlett. 704, (2007). 
A. P. M. Marcos, B. Paulo, M. Pablo, B. Sergio, M. Sidnei, Z. Nilo, G. B. Helio, F. C. F. Alex, J. Braz. Chem. Soc. 17, 408, (2006).

G. W. Kabalka, M. Al-Masum, Org. Lett. 8, 11, (2006).

M.Shanmugasundaram, A. L. Aguirre, M. Leyva, B. Quan, L. E. Martinez, Tetrahedron Lett. 48, 7698, (2007).

(a) D. Adam, Nature, 421, 571, (2003), (b). C. O. Kapper, Angew. Chem. Int. Ed. 43, 6250, (2004).

Z. Ni, R. I. Masel, J. Am. Chem. Soc. 128, 12394, (2006).
E. F. DiMauro, J. M. Kennedy, J. Org. Chem. 72, 1013, (2007).

J.-X. Wang, X. N. Shi, X. Q. Men, L. B.Zhao, Synth. Commun. 33, 2849, (2003).

X. Q. Huang, H. X. Li, J.-X. Wang, X. F. Jia, Chin. Chem. Lett. 16, 607, (2005).

A. T. Balaban, Tetrahedron, 26, 739, (1970).

F. S. Al-Hajjar, S. S. Sabri, J. Heterocyclic Chem. 23, 727, (1986),

T. C. Sharma, H. Patel, M. M. Bokadia, Indian J. Chem. 11, 703, (1973). 